\title{
EVALUATION OF THE QUALITY OF LIFE IN THE CZECH ADMINISTRATIVE REGIONS
}

*Note: Do not include the author(s) names and information as this document will be blind reviewed and they will be entered during proposal submission.

\begin{abstract}
Measuring the quality of life of given units (cities, urban regions, countries etc.) is the task that is quite often discussed by researchers and independent organizations. The result of the analysis usually leads to a composite index that allows ranking of the units. The problem itself is multiple criteria decision analysis (MCDA) problem. This kind of problems is often solved by simple approaches that need not lead always to correct results. The main aim of the paper is to develop an AHP model with absolute measurement for evaluation of quality of life in 14 administrative regions in the Czech Republic based on 3 main groups of totally 24 criteria and compare its results with official methodology. This methodology is based on equal importance of all criteria and a quite unacceptable MCDA technique is applied. The results given by the AHP model are compared to the ones derived by official methodology, by several MCDA methods (SAW, TOPSIS, PROMETHEE), and by a data envelopment analysis model without explicit inputs.
\end{abstract}

Keywords: analytic hierarchy process, quality of life, composite index, data envelopment analysis.

\section{Introduction}

In many areas of human life, there are quite often defined composite indices with the aim to measure different aspects of development of countries, regions, cities, etc. On the country level, among the well-known indices belong Human Development Index, Multidimensional Poverty Index and Gender Inequality Index defined by United Nations Development Programme (UNDP), Happy Planet Index, Human Capital Investment Index, and many more. Similar indices are proposed in urban or regional levels. They are often defined as multi-dimensional indicators for measurement of quality of life.

\section{Literature review}

There are many papers dealing with analysis of regional quality of life using various MCDA models including AHP. Lotfi and Soleimani (2009) propose a general framework for objective evaluation of quality of life in north Iran. Feneri et al. (2015) discuss the 
measuring the quality of life in Thessaloniki (Greece) urban region and as one of the modelling tools use the AHP. Information about applications of the AHP not only in regional analyses can be found in (Vaidya and Kumar, 2006).

\section{Objectives}

The main aim of the paper is to discuss and re-define the one of the indicators for evaluation of quality of life in 14 administrative regions in the Czech Republic based on 24 criteria in 3 main groups. The official methodology works with equal weights of criteria, which is hardly acceptable.

\section{Research methodology}

AHP model is built in order to solve the defined problem. The model tries to derive an objective indicator based on several subjective opinions, i.e. constructs the final composite index as the aggregation of several partial indices. The proposed AHP model combines relative and absolute measurement. The weights of the criteria are derived using relative measurement, the final indices of the regions using absolute measurement. In addition to the AHP model, possibilities of application of a DEA model without explicit inputs are analyzed.

\section{Data analysis}

The data set for the analysis contains 14 alternatives (administrative regions) and 24 cardinal criteria in 3 main groups. The first group of criteria takes into account conditions for a healthy and long life. The second group describes living standard in the region and the last one is a group of criteria for a healthy environment and sustainable development within the region.

\section{Limitations}

It is clear that any composite index cannot be never fully objective. The evaluation of quality of life is mostly influenced by individual preferences of decision makers. Other limitations are given by the size of regions under evaluation. They are not homogenous and the quality of life in particular parts may differ significantly.

\section{Conclusions}

Quality of life evaluation is an interesting MCDA problem and its correct solving requires a combination of subjective and objective factors. The paper proposes a groupdecision making approach that combines AHP with relative and absolute measurement and finally derives final composite indices for the regions under evaluation.

\section{Acknowledgements}


ISAHP Article: A Style Guide for Paper Proposals To Be Submitted to the International Symposium on the Analytic Hierarchy Process 2016, London, U.K.

The research is supported by the Czech Science Foundation - project no. 16-01821S.

\section{Key References}

Feneri, A.M., Vagiona, D., \& Karanikolas, N. (2015). Multi-criteria decision making to measure quality of life: An integrated approach for implementation in the urban area of Thessaloniki, Greece. Applied Research in Quality of Life, 10(4), 573-587.

Lotfi, S., \& Solaimani, K. (2009). An assessment of urban quality of life by using analytic hierarchy process approach. Journal of Social Sciences, 5(2), 123-133.

Saaty, T.L. (1990). The Analytic Hierarchy Process. Pittsburgh, RWS Publications.

Vaidya, O.S., \& Kumar, S. (2006). Analytic hierarchy process: An overview of applications. European Journal of Operations Research, 169(1), 1-29. 